\section{Eruptive pseudoangiomatosis in adults with immune system disorders: A report of two cases}

\author{
Sandra González Saldaña, ${ }^{1}$ \\ Raul G. Mendez Flores, ${ }^{1}$ \\ Andrea F. López Gutiérrez, ${ }^{1}$ \\ Lupita N. Salas Núñez, ${ }^{1}$ \\ Ana Karen Hermosillo Loya, ${ }^{1}$ \\ Marisol Ramírez Padilla, ${ }^{1}$ \\ Mercedes Hernández Torres ${ }^{2}$ \\ ${ }^{1}$ Department of Dermatology, Civil \\ Hospital of Guadalajara, Jalisco; \\ ${ }^{2}$ Private Practice Dermatopathology, \\ Guadalajara, Mexico
}

\begin{abstract}
Eruptive pseudoangiomatosis is a cutaneous disease of unknown origin, characterized by the sudden appearance of small, asymptomatic angioma-like erythematous papules surrounded by a pale halo that resolves spontaneously. It occurs due to transitory dermal blood vessel dilation and has been associated with viral infections and hematologic disorders. To this day, it remains a disease that affects mostly the lower spectrum of life. Most of the cases have been reported in children and few reports have described its association with immune system disorders. The diagnosis is based upon clinical presentation. Histopathological analysis has no pathognomonic findings and can include dilated dermal blood vessels, perivascular lymphocytic infiltration and plump endothelial cells. We report a 21-years-old female diagnosed with HIV in AIDS stage and a 19years-old male diagnosed with nonHodgkin's lymphoma.
\end{abstract}

\section{Introduction}

Eruptive pseudoangiomatosis is a cutaneous disease of unknown origin, characterized by the sudden appearance of small, asymptomatic angioma-like erythematous papules surrounded by a pale halo that resolves spontaneously. It occurs due to transitory dermal blood vessel dilation ${ }^{1}$ and has been associated with viral infections and hematologic disorders. ${ }^{2}$ Most of the cases have been reported in children and few reports had described its association with with immune system disorders. Hereby, we report two cases in young adults. The objectives of these case reports are to bring awareness to this condition in order to facilitate its correct diagnosis as well as to provide reassurance to the patient.

\section{Case 1}

A 21-years-old female presented to our clinic with a fifteen-day history of skin lesions. Initially, she noticed erythema and pruriginous papules in her abdomen. Extension to her face, back, upper and lower extremities was noted sequentially. The patient also had a fifteen-day history of non-productive cough, odynophagia and significant weight loss.

Skin examination revealed multiple, disseminated, symmetrically distributed 2-4 $\mathrm{mm}$ erythematous papules with diffuse borders. They affected the face, thorax, abdomen, upper extremities and lower extremities (Figure 1a). Dermoscopy showed complete blanching when pressure was applied (Figure 2). Oral mucosa examination revealed multiple raised white patches surrounded with erythema in multiple sites of the oral cavity. Hair and nails were unaffected.

Work up included a complete blood count and HIV, HBV and HCV serologies. An incisional biopsy was taken for histopathological analysis. The hematoxylin and eosin staining revealed a normal epidermis and dilated blood vessels in the dermis. She was diagnosed with HIV in AIDS stage (Viral load 71,300 RNA copies/mL and CD4+ count of <200), pneumocystis jirovecii pneumoniae and eruptive pseudoangiomatosis. Skin lesions started to fade in the third week of hospitalization.

\section{Case 2}

A 19-years-old male presented to our clinic with a seven-day history of skin lesions on the face, upper and lower extremities. The patient had been recently diagnosed non-Hodgkin's lymphoma.

Skin examination revealed multiple, disseminated, symmetrically distributed 1-3 $\mathrm{mm}$ erythematous papules. They affected predominantly lower extremities but were also present in the face and upper extremities (Figure 1b). The lesions completely blanched with pressure and rapidly refilled when released. Oral mucosa, hair and nails were unaffected.

Serological tests for HIV, HBV, HCV, Epstein-Barr virus and cytomegalovirus were all negative. An incisional biopsy was taken for histopathological analysis. The
Correspondence: Raul Gerardo Mendez Flores, M.D. Department of Dermatology, Civil Hospital of Guadalajara Av. Hospital 278, Sector Hidalgo, CP 44280, Guadalajara, Jalisco Mexico

Tel.: 011-52-6144881440.

E-mail: mendezfloresdr@gmail.com

Key words: Eruptive pseudoangiomatosis, immune system disorders, HIV, non-hodgkin lymphoma.

Conflict of interest: The author declare no potential conflict of interest.

Received for publication: 12 August 2020. Accepted for publication: 10 OCtober 2020 .

This work is licensed under a Creative Commons Attribution-NonCommercial 4.0 International License (CC BY-NC 4.0).

${ }^{\circ}$ Copyright: the Author(s), 2020

Licensee PAGEPress, Italy

Dermatology Reports 2020; 12:8836

doi:10.4081/dr.2020.8836

hematoxylin and eosin staining revealed a normal epidermis, dermal edema, and dilated blood vessels with plump endothelial cells surrounded by a mild perivascular lymphocytic infiltrate (Figure 3).

The histopathological report was consistent with eruptive pseudoangiomatosis. No treatment was prescribed, and complete resolution occurred in 12 days.

\section{Discussion}

Eruptive pseudoangiomatosis is a rare cutaneous pathology characterized by erythematous angioma-like papules that resolve spontaneously. The first cases were described in 1969 by Cherry et al. and it was first named by Prose et al. in 1993. . $^{3,4}$ For the first thirty-one years of the disease, cases were only detected in children. It was not until the early 2000s that the first cases were reported in adults. ${ }^{5,6}$ To this day, it remains a disease that affects mostly the lower spectrum of life.

Its association to viral infections has been suspected, and some authors consider it to be a paraviral exanthem ${ }^{7}$ due to its clinical course with spontaneous remission. Regardless, no single microbe etiology has been identified. There have been reports linking this dermatosis to Culex pipiens mosquito bite, CMV, ECHO virus, EpsteinBarr virus, adenovirus infections; and lymphoblastic leukemia. ${ }^{8}$ In adult patients, it is also strongly associated with immunocom- 
promising conditions, such as the aforementioned hematological malignancies, kidney transplantation or elderly patients. It is of note that in 2009, Hermida et al. reported one of the few cases in the literature regarding an HIV+ patient. $^{2}$

Eruptive Pseudoangiomatosis is usually asymptomatic, but $22 \%$ of patients can also manifest pruritus. ${ }^{9}$ Normally, the lesions resolve without treatment in fifteen days. Relapses have also been reported in a small percentage of patients. ${ }^{9}$ There are some differences between the clinical presentation in children and adults. In adults, the prodro- mal phase is normally non-existing, and the clinical resolution is longer (more than one month). Hermida et al. also reported a higher prevalence in adult women than in adult men. ${ }^{2}$ Although the diagnosis of eruptive pseudoangiomatosis is established based on clinical findings, incisional biopsies were taken for study purposes. Written informed consent was obtained from all patients. Histopathological analysis has no pathognomonic findings and can include dilated dermal blood vessels, perivascular lymphocytic infiltration in the superficial capillary plexus and plump endothelial cells. The precise cause of these changes has not been elucidated, but it has been theorized that it might be a dermal hypersensitivity reaction to a viral particle or a direct viral effect on the vascular endothelium. ${ }^{10}$ There are no specific laboratory findings. No specific treatment is required and lesions usually resolve spontaneously in one to two weeks. ${ }^{9}$ In conclusion, Eruptive pseudoangiomatosis is a pathology not widely known and probably underdiagnosed due to the fact that it is self-limited. More studies are needed to establish a known etiology and associations.

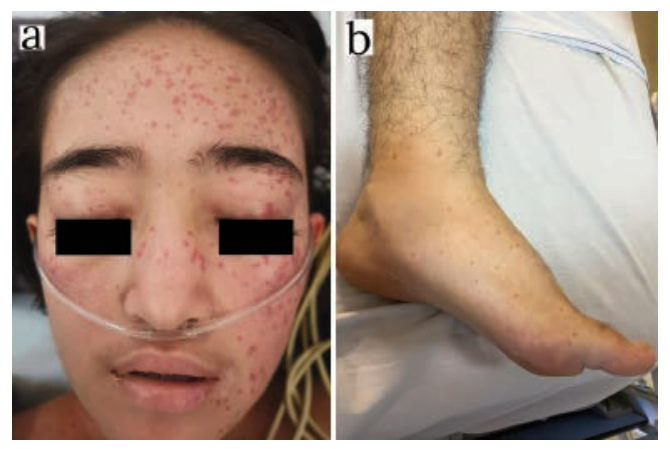

Figure 1. a) Angioma-like papules in a 21-year-old HIV-positive woman; b) Pinpoint erythematous papules with a pale halo in a 19-year-old male with non-Hodgkin lymphoma.

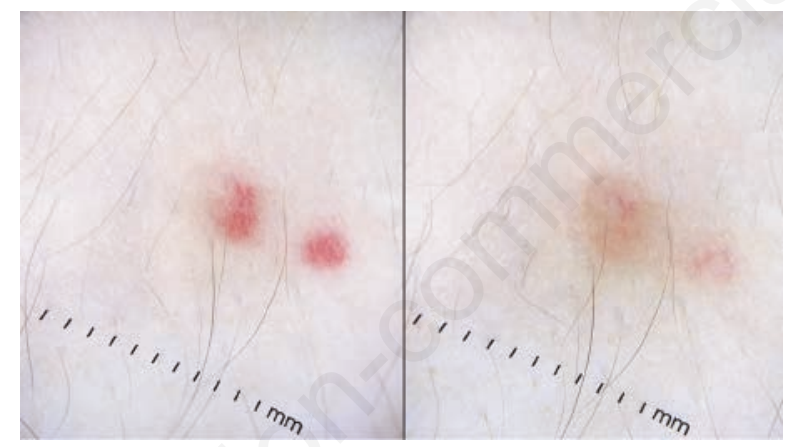

Figure 2. Dermoscopic images of eruptive pseudoangiomatosis showing blanching under pressure (Dermlite DL4W, California, USA).

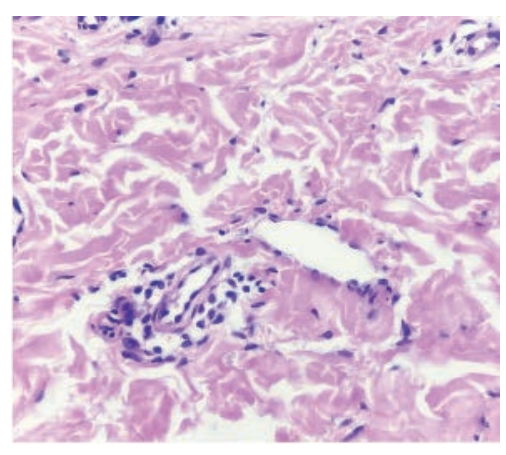

Figure 3. Hematoxylin-eosin stain showing edema and dilated capillaries with prominent, plump endothelial cell lining and perivascular lymphocytic infiltrate $(40 \mathrm{X})$.

\section{References}

1. Yang JH, Kim JW, Park HS, et al. Eruptive pseudoangiomatosis. J Dermatol 2006;33:873-6.

2. Hermida MD, Della Giovanna P, García $\mathrm{S}$, et al. Pseudoangiomatosis eruptiva del adulto. Reporte de tres casos, dos en inmunosuprimidos. Med Cutan Iber Lat Am 2009;37:147-50.

3. Cherry JD, Bobinski JE, Horvath FL, Comeri GD. Acute hemangioma-like lesions associated with ECHO viral infections. Pediatrics 1969;44:498-502.

4. Prose NS, Tope W, Miller SE, Kamino H. Eruptive pseudoangiomatosis: A unique childhood exanthem? J Am Acad Dermatol 1993;29:857-9.

5. Navarro V, Molina I, Montesinos E, et al. Eruptive pseudoangiomatosis in an adult. Int J Dermatol 2000;39:237-8.

6. Guillot B, Dandurand M. Eruptive pseudoangiomatosis arising in adulthood: 9 cases. Eur J Dermatol 2000;10:455-8.

7. Chuh A, Panzer R, Rosenthal AC, et al. Annular eruptive pseudoangiomatosis and adenovirus infection: a novel clinical variant of paraviral exanthems and a novel virus association. Acta Derm Venereol 2017;97:354-7.

8. Venturi C, Zendri E, Medici MC, et al. Eruptive pseudoangiomatosis in adults: a community outbreak. Arch Dermatol 2004;140:757-8.

9. Kim JE, Kim BJ, Park HJ, et al. Clinicopathologic review of eruptive pseudoangiomatosis in Korean adults: report of 32 cases. Int $\mathrm{J}$ Dermatol 2013;52:41-5.

10. Chopra D, Sharma A, Kaur S, Singh R. Eruptive pseudoangiomatosis - cherry angiomas with perilesional halo. Indian J Dermatol Venereol Leprol 2018;84: 424-30. 